* Corresponding author

Phone +420377632300

E-mail address:las@kme.zcu.cz (V. Laš)

Article information

Article history: AMS-Volume15-No.1-00102-11

Received 4 April 2011

Accepted 15 May 2011

Available online 26 August

\section{Impact Force Reconstruction for Structural Health Monitoring of Composite Beam}

\author{
Vladislav Laš a* , Tomáš Kroupa b , Jan Bartošek c, Robert Zemčík ${ }^{d}$ \\ a Department of Mechanics, Faculty of Applied Sciences, University of West Bohemia, Univerzitní 22, 30614 Plzeň, (zech Republic \\ ${ }^{b}$ Department of Mechanics, Faculty of Applied Sciences, University of West Bohemia, Univerzitni 22, 30614 Plzeň, (zech Republic \\ 'Department of Mechanics, Faculty of Applied Sciences, University of West Bohemia, Univerzitní 22, 30614 Plzeň, Czech Republic \\ ${ }^{d}$ Department of Mechanics, Faculty of Applied Sciences, University of West Bohemia, Univerzitní 22, 30614 Plzen̆, Zzech Republic
}

\section{BIOGRAPHICAL NOTES}

prof. Ing. Vladislav Laš, CSc. (born 1952) graduated at the University of Mechanical and Electrical Engineering in Pilsen (1977). In 1983 he defended thesis in the field of "Tooth deformation of bevel and hypoid gears". In 1989 he became associate professor and in 2008 professor at the branch of Mechanics of Materials. From 2005 till now he is the head of the Department of Mechanics at the Faculty of Applied Sciences at the University of West Bohemia (UWB) in Pilsen. From 2006 till now he is a member of the Committee of the Czech Society for Mechanics. The fields of his scientific activity are mechanics of composite materials, damage mechanics and structural health monitoring. $\mathrm{He}$ is author or co-author of 30 papers in journals and about 180 papers in conference proceedings.

Ing. Tomáš Kroupa, PhD. (born 1980) graduated from the University of West Bohemia in Pilsen in 2003 and received the Ing. degree in the field of Mathematical-physical modeling. He was granted the Ph.D. degree after successful defence of thesis with title Damage of composites due to impact in 2006. His research is aimed at finite element modeling of composite materials. He has participated on several grant projects from various agencies, e.g., Design of smart dynamically loaded structures (GAČR P101/11/0288, 2011 2014), Stress propagation and degradation processes in anisotropic laminates (GA AV ČR IAA200760611, 2006 - 2010), Prediction of failures of heterogenous materials, components of mechanical and biomechanical systems (MŠMT no. MSM 4977751303 2008, 2005 - 2009). He has worked as a research fellow at the University of West Bohemia in Pilsen, Department of mechanics and Department of machine design since 2006 and 2010, respectively. He was also employed at the Academy of Sciences of Czech Republic, Institute of Thermomechanics from 2005 until 2010. He is the author and co-author of approx. 50 papers.

Ing. Jan Bartošek (born 1985) graduated from the University of West Bohemia in Pilsen with Bc. degree in 2007 and with Ing. degree in 2009. His field of study was Machine design and his diploma thesis was named Design of composite bicycle frame. His thesis was awarded as the most interesting one by a representative of the MBtech company on a student conference of UWB in Pilsen in 2009. He started his doctoral studies on Department of Mechanics on the University of West Bohemia in Pilsen in 2009. His research is aimed at monitoring of composite structures and at finite element modelling. He is the author or co-author of several papers.

Ing. Robert Zemčík, PhD. (born 1977) studied at the University of West Bohemia in 
Pilsen and received the Ing. degree in 2000 (Applied sciences and informatics) and the Ph.D. degree in 2005 (Non-stationary progressive failure analysis of fiber-reinforced composites). He has worked as a research fellow at UWB, Department of mechanics since 2005. He also worked at DLR (German Aerospace Centre, Braunschweig) in 2003/2004 on a project: Development of shell finite elements with embedded active materials. His research is aimed at finite element modeling of composite materials, piezoelectric materials, and stereophotogrammetry. He has participated on 11 grant projects from various agencies and he was the principal investigator of one GACR project. He is the author or co-author of 4 papers in impacted journals (with 9 citations), 14 papers in other journals, and more than 47 papers in conference proceedings.

\section{KEYWORDS}

Unidirectional Composite Wide Beam, Identification, Impact Location, Impact Force Reconstruction

\section{ABSTRACT}

Reconstruction of the impact force history and identification of the impact location are important tasks in structural health monitoring (SHM) applications, namely in case of composite structures. These tasks are critical especially in cases, when it is not possible to measure the impact force directly and the knowledge of the intensity and position of loading force is necessary for the damage prediction of the structure. This paper tests two methods for the reconstruction of impact force and identification of impact location. The first method uses finite element analysis (FEA) for determination of a transfer matrix of a system. The second method uses experimental results only. The methods are tested on an orthotropic prismatic wide beam with rectangular cross-section. Three piezoelectric patch transducers are bonded to the beam bottom-surface to measure deformations.

\section{Introduction}

Composite materials, namely carbon fiber reinforced epoxy laminates, are widely used thanks to their high strength and stiffness to weight ratios. Nevertheless, the design process of structures which contain parts from composite materials is complicated due to effects such as non-linear behavior (Kroupa et al., 2011), specific damage behavior (Laš et al., 2008), and directional dependence of velocity of propagation of stress waves (Červ et al., 2010). Furthemore, composites are sensi- tive to transverse loading, which can cause delamination and cracks in matrix and thus significantly reduce the stiffness or strength of the construction. These defects can be invisible to surface inspection. Therefore, they are currently detected by non-destructive techniques like ultrasonic, $\mathrm{X}$-ray or coin tapping, which are time and cost consuming and require the construction to be taken out of service. In contrary, the condition of construction can be evaluated during operation from measurements of sensors placed over the structure. This principle is so-called structural health monitoring. The identification of impact force and impact location is an important task of such systems and the ideal identification method should identify the impact force, or even the combination of impact forces, on complex structures in real time with low dependence of operating noise.

The impact identification problems have been studied by many researchers in recent years and several methods were proposed. The often used one is the inversion of forward problem, which can be performed in time, frequency or spectral domain. Direct deconvolution is a well-known ill-conditioned problem and its results are strongly influenced by quality of experimental data, appropriateness of the mechanical model and robustness of employed algorithm. Many researches define the problem rather as a minimization of the difference between measured and modelled responses of the impacted structure. Additional terms and constraints are added to minimization to regulate oscillations in results.

(Jacquelin et al., 2003) analyzed the deconvolution in time domain. The influence of sensors location and different regularization methods were investigated. Similarly, (Gunawan et al., 2006) used the time domain. The impact force was approximated by cubic spline and the two-step B-spline regularization method was developed. On the other hand Yan and Zhou (Yan et al., 2009) used Chebyshev polynomials to represent the impact force and the modified genetic algorithm to solve the minimization problem.

(Park et al., 2005) determined the system experimentally and investigated several types of impacts. (Martin et al., 1996) used the Fast Fourier Transform to switch into frequency domain and solved the deconvolution directly. Furthermore (Doyle, 1997) employed the wavelet deconvolution and modelling with FEA. Other researches preferred to work in spectral domain. (Hu et al., 2007) formulated the minimization with regularization parameter and constraint, which was solved by 
quadratic programming method. Moreover, different type of sensors were compared and Chebyshev polynomials were employed to reduce the number of unknowns. (Atobe et al., 2009) used the gradient projection method to solve the minimization problem and compared the determination of the system by experiment or by FEA. Finally (Sekine et al., 2009) formulated the minimization where multiple impacts can be identified. Another possibility is to define the minimization in recursive form in time domain and to use filtering method to solve the investigated problem. (Seydel et al., 2001) used smoothing-filter method and investigated the influence of sensor locations and boundary conditions. Similarly, (Zhang et al., 2008) implemented smoothing-filter algorithm with the possibility of realtime computations.

The location of impact within these methods is often estimated from the minimization of the error between measured and modelled responses along the structure. This can be done by direct search methods (Hu et al., 2007) or by some other optimization techniques (Atobe et al., 2009). Another possibility is to use the techniques derived from methods used in acoustic emission (AE) (Kudu et al., 2008; Seydel et al., 2001), where the difference in arrival time of signal is determined and the location of impact is estimated from velocity of waves. Unfortunately, the determination of exact time of arrival in composite material or complex structures is limited because of the dispersion and reflexion of waves on boundaries. The alternative is calculation of distribution of energy in defined time step and the determination of its maximum (Park et al., 2005).

Totally different approach is the determination of impact force and force location from models based on neural networks (LeClerc et al., 2007). The model is composed of parallel elements connected by defined relations and trained by preliminary tests. The output of the model is then set by learned behaviour. The weakness of such approach is the necessity of learning period and uncertain reaction of model to not learned impacts.

The above cited papers differ in several features like complexness of geometry, determination of the system model or the type and number of sensors. The impact force was investigated on metal beam (Martin et al., 1996), metal plate (Doyle, 1997; Jacquelin et al., 2003; Gunawan et al., 2006), composite plate (Kudu et al., 2008; Hu et al., 2007) or stiffened composite plate (Seydel et al., 2001; Park et al., 2005; Zhang et al., 2008;
Hu et al., 2007; Yan et al., 2009; Atobe et al., 2009; Sekine et al., 2009). The model of the system is defined analytically (Seydel et al., 2001; Zhang et al., 2008; Yan et al., 2009) or determined by FEA (Gunawan et al., 2006; Hu et al., 2007; Atobe et al., 2009; Sekine et al., 2009) or by experiment (Park et al., 2005; Atobe et al., 2009). Signal is mostly obtained from strain gagues (Atobe et al., 2009; Sekine et al., 2009), accelometers (Martin et al., 1996; Doyle, 1997; Hu et al., 2007), simple piezoelectric sensors (Hu et al., 2007) or from sensor network (Park et al., 2005; Yan et al., 2009).

\section{Problem Formulation}

The main goal of the paper is to identify the impact location and to reconstruct the impact force on the unidirectional carbon/epoxy composite wide beam. Three piezoelectric transducers are used for measuring the response. Both, the response and the loading force are measured in time interval described by time vector

$\mathbf{t}=\left[t_{k}\right], \quad k=1 \ldots K$

The response (strain) from the sensor $s$ and the loading force (impact force) are considered as time dependent vectors

$\varepsilon^{s}=\left[\varepsilon^{s}\left(t_{k}\right)\right]$

and

$\mathbf{F}=\left[F\left(t_{k}\right)\right]$.

The whole response vector for three sensors has the form

$\varepsilon=\left[\begin{array}{l}\varepsilon^{1} \\ \varepsilon^{2} \\ \varepsilon^{3}\end{array}\right]$.

In general, the relation between the loading force and the responses of the system can be described as

$\varepsilon=\mathrm{GF}$

where $\mathbf{G}$ is the transfer matrix in form

$\mathbf{G}=\left[\begin{array}{cccc}g\left(t_{1}\right) & 0 & \cdots & 0 \\ g\left(t_{2}\right) & g\left(t_{1}\right) & \cdots & 0 \\ \vdots & \vdots & \ddots & \vdots \\ g\left(t_{K}\right) & g\left(t_{K}\right) & \cdots & g\left(t_{1}\right)\end{array}\right]$. 
Dimensions of the beam and piezo patches are shown in Fig. 1.

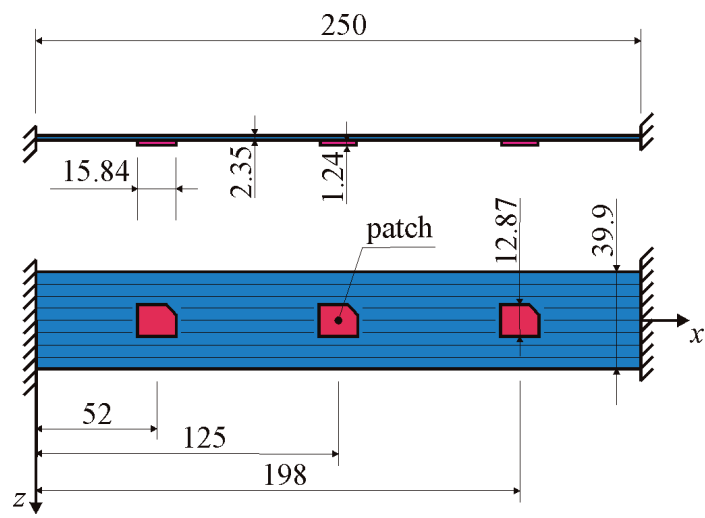

Fig. 1: Dimensions of the beam with piezoelectric patch transducers and schematically depicted fibers.

\section{Experiment}

Experiment was performed using the equipment shown in Fig. 2. Piezoelectric patch transducers were used for measuring the strains in three locations. Impact hammer Brüel \& Kjær type 8202 was used for loading and measuring the impact force.

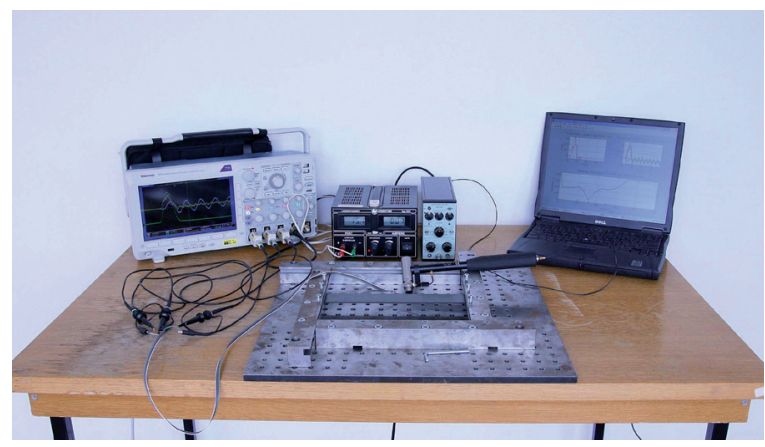

Fig. 2: Experimental setup.

\section{Determination of Transfer Matrix using FEA}

The first method for the determination of the transfer matrix uses FEA. The advantage of this method is that the main part of the process can be fully automated. The disadvantage is the necessity of developing of the FE model, results of which should correspond to the experimental data as much as possible.

\subsection{Finite element model}

FE model is built using parametric procedures in MSC.Marc/Mentat software using four node shell ele- ments (Fig. 3). Two sets of the material properties (orthotropic) are used. One for pure composite part and one for parts with piezo patches attached (see Tab. 1).

Table 1 Material parameters.

\begin{tabular}{|c|c|c|}
\hline Parameter & Composite & $\begin{array}{c}\text { Composite with } \\
\text { patches }\end{array}$ \\
\hline$E_{1}[\mathrm{GPa}]$ & 130.0 & 118.2 \\
\hline$E_{2}[\mathrm{GPa}]$ & 10.5 & 15.1 \\
\hline$G_{12}[\mathrm{GPa}]$ & 3.8 & 4.5 \\
\hline$\nu_{12}[-]$ & 0.3 & 0.3 \\
\hline$\rho 12\left[\mathrm{~kg} \cdot \mathrm{m}^{-3}\right]$ & 1500.0 & 2465.0 \\
\hline
\end{tabular}

The response measured by piezo patch $s$ corresponds to quantity which can be calculated as:

$\varepsilon^{s}=\frac{h}{2 l_{0}}\left[\left(\varphi_{z}^{2}-\varphi_{z}^{1}\right)+\left(\varphi_{x}^{3}-\varphi_{x}^{4}\right)\right]$

where $h$ is the thickness of the beam, $l_{0}=10 \mathrm{~mm}$ is the dimension of patch active area and $\varphi$ are time dependent vectors of rotations around corresponding axis taken from nodes in the middle of both opposing edges of the patch.

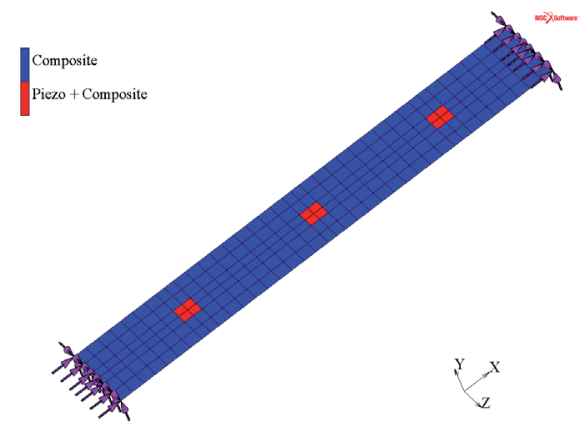

Fig. 3: Finite element model with boundary conditions and piezo patches.

\subsection{Transfer matrix}

Loading force for given impact location is reconstructed using so-called base loading functions

$\mathrm{F}=\Phi \mathrm{R}$

where

$\mathbf{R}=\left[R_{k}\right]$

is a vector of coefficients and 


$$
\Phi=\left[\Phi_{k}\right]
$$

is matrix of the base loading functions where

$$
\Phi_{i}=\left[\varphi_{i}\left(t_{k}\right)\right] .
$$

The base loading functions are all the same ramp function mutually shifted in time by time step $\Delta t=t_{k+1}-t_{k}$. Fig. 4 shows the shapes of the base loading functions.

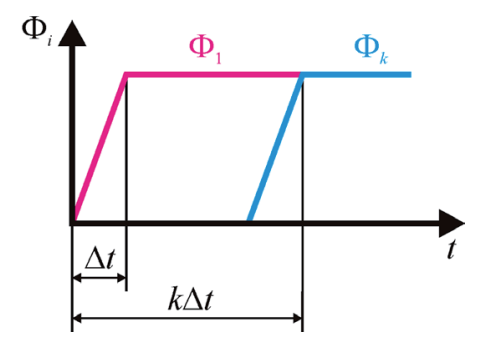

Fig. 4: Base loading functions.

Response vectors are reconstructed as

$\varepsilon=\Psi \mathrm{R}=\left[\begin{array}{c}\Psi^{1} \\ \Psi^{2} \\ \Psi^{3}\end{array}\right] \mathrm{R}$

where

$\Psi^{s}=\left[\Psi_{k}^{s}\right]$

is the matrix of base response functions for sensor $s$. Thanks to the choice of $\Phi_{i}$ the base response functions are again all the same just shifted in time. The base response function

$\Psi_{i}^{s}=\left[\psi_{i}^{s}\left(t_{k}\right)\right]$

is the response to base loading function $\Phi_{i}$ for sensor $s$.

The vector of coefficients then can be expressed from relation (8) as

$\mathrm{R}=\Phi^{-1} \mathrm{~F}$.

Using equation (12) we get

$\varepsilon=\Psi \Phi^{-1} \mathrm{~F}$

which gives us the transfer matrix in (5)
$\mathrm{G}^{\mathrm{FEA}}=\Psi \Phi^{-1}$.

\section{Determination of Transfer Matrix using Experimental Data}

Transfer matrix for given impact location is determined from a set of impact tests using the least square approximation

$\left[\begin{array}{c}\mathbf{F}_{i m p}^{1} \\ \vdots \\ \mathbf{F}_{i m p}^{I}\end{array}\right] \mathbf{g}=\left[\begin{array}{c}\varepsilon_{i m p}^{1} \\ \vdots \\ \varepsilon_{i m p}^{I}\end{array}\right]$

where $I$ is the number of impact tests performed, $\mathbf{g}$ is the vector of coefficients of the transfer matrix

$\mathbf{g}=\left[g\left(t_{k}\right)\right]$

$\mathbf{F}_{i m p}^{i}$ is the matrix of impact loading vectors

$\mathbf{F}_{\text {imp }}^{i}=\left[\begin{array}{cccc}F\left(t_{1}\right) & 0 & \cdots & 0 \\ F\left(t_{2}\right) & F\left(t_{1}\right) & \cdots & 0 \\ \vdots & \vdots & \ddots & \vdots \\ F\left(t_{K}\right) & F\left(t_{K}\right) & \cdots & F\left(t_{1}\right)\end{array}\right]$

and $\boldsymbol{\varepsilon}_{\mathrm{imp}}$ is the response vector for $\boldsymbol{i}$-th impact test. The matrix $G^{\text {REAL }}$ is determined using vector $\mathbf{g}$, which is placed into matrix $\mathrm{G}$ in a similar fashion as shown in equation (6).

\section{Methodology of Identification of Impact Location}

Firstly, the impact forces are calculated from the measured responses $\varepsilon_{\text {exp }}$ using the least square method with restriction to calculate only non-negative force values

$\mathbf{G}\left(x_{n}\right) \mathbf{F}\left(x_{n}\right)=\varepsilon_{\text {exp }}$ for $n=1 \ldots N$.

$F\left(t_{k}\right) \geq 0$ for $k=1 \ldots K$.

This is performed for all possible impact locations $N$. Symbol $x_{n}$ denotes the location of a node (FEA) or an impact test position (REAL).

Once the impact forces are known, the estimated impact location $x_{e}$ is chosen as the one, which satisfied the condition 


$$
\begin{aligned}
& \left\|\varepsilon_{\text {exp }}-\mathbf{G}\left(x_{e}\right) \mathbf{F}\left(x_{e}\right)\right\|^{2} \\
& =\min _{n}\left(\left\|\varepsilon_{\text {exp }}-\mathbf{G}\left(x_{n}\right) \mathbf{F}\left(x_{n}\right)\right\|^{2}\right) .
\end{aligned}
$$

An extrapolation is performed subsequently between two impact locations adjacent to $x_{\mathrm{e}}$ using linear approximation functions

$$
\begin{aligned}
& h_{1}=\frac{1}{2}(1-p) \\
& h_{2}=\frac{1}{2}(1+p)
\end{aligned} \quad p \in\langle-1,1\rangle .
$$

The transfer matrix for given parameter $p$ is then calculated as

$$
\mathbf{G}(p)=h_{1}(p) \mathbf{G}\left(x_{n}\right)+h_{2}(p) \mathbf{G}\left(x_{n+1}\right) .
$$

The location for given parameter $p$ can be calculated as

$x=h_{1}(p) x_{n}+h_{2}(p) x_{n+1}$.

The final identification is performed in the same way as the estimation, but using smaller range and finer resolution.

\section{Results}

Typical results from the identification are shown in Fig 5 and 6 for real impact location $x=162 \mathrm{~mm}$. The real, estimated and identified impact forces are shown in both figures in bottom position. The time dependencies of strains from piezoelectric patches are shown in the middle position. The top graphs display residuals

$r\left(x_{n}\right)=\left\|\varepsilon_{\exp }-\mathbf{G}\left(x_{n}\right) \mathbf{F}\left(x_{n}\right)\right\|^{2}$.

The identification performed using the experimental impact data proves to be more precise in the prediction of both the impact force and the location.

The results obtained from almost all other tested impact locations show similar precision as the ones shown in Fig. 5 and 6. Problems arise when the impact location is close to the outer patches in case of the identification using FEA. The FEA model can not describe properly the response of the piezoelectric patches.

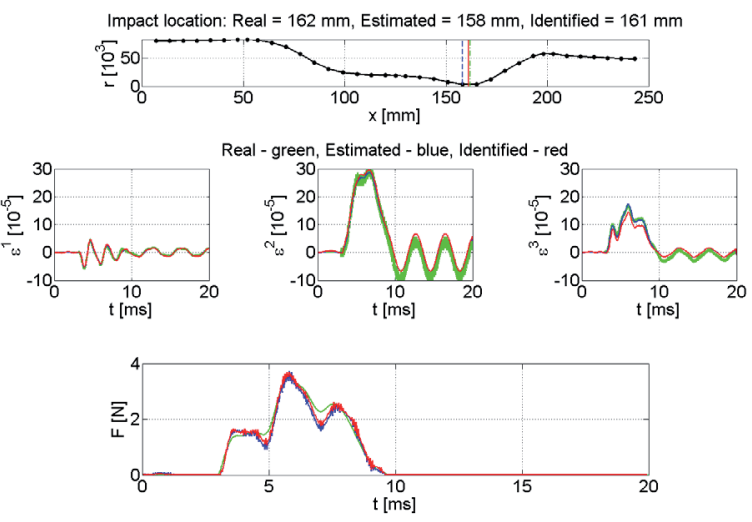

Fig. 5: Results from identification using FEA analysis.
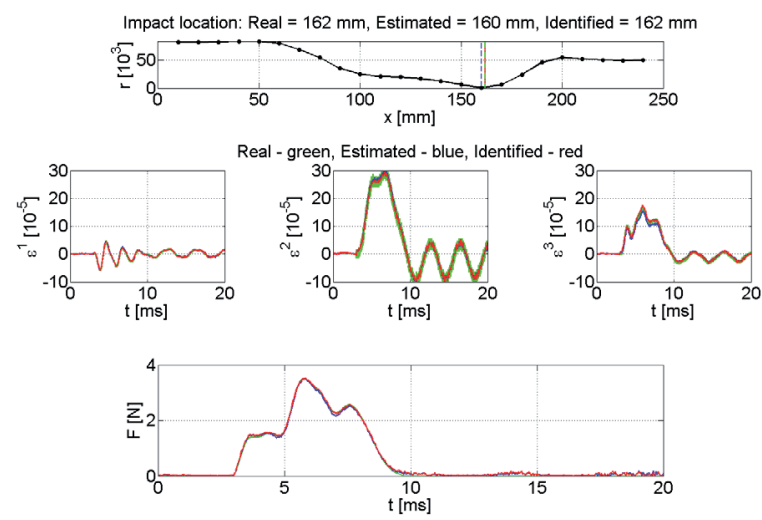

Fig. 6: Results from identification using REAL experimental data.

\section{Conclusion}

Two approaches for the reconstruction of the impact force and identification of the impact location were tested in the paper. The first method used experimental data for the determination of the transfer matrix of the system and it proved to be more precise but requires much more measurements than the second method based on finite element analysis.

The usage of the finite element analysis was tested with the intention of performing subsequent failure analysis. Nevertheless, it requires better description of the patches for example using fully coupled piezoelectric material model. Nevertheless, both approaches proved to be capable of performing the identification process with acceptable results.

Determination of suitable sensor distribution on one or two dimensional problem of a composite plate or more complex structure will be the aim of the future work. 


\section{Acknowledgement}

The work has been supported by the research project of the Ministry of Education of Czech Republic MSM 4977751303 and the project of the Grant agency of the Czech Republic no. P101/11/0288.

\section{References}

Atobe, S., Kuno, S., Hu, N., Fukunaga, H. (2009). Identification of Impact Force on Stiffened Composite Panels. Transactions of Space Technology Japan, vol. 7, pp. 1-5

Červ, J., Kroupa, T., Trnka, J. (2010). Influence of principal material directionsof thin orthotropic structures on Rayleigh-edge wave velocity. Composite structures, vol. 92, pp. 568-577

Doyle, J.F. (1997). A wavelet deconvolution method for impact force identification. Experimental Mechanics, vol. 37, pp. 403-408

Gunawan, F.E., Homma, H., Kanto, Y. (2006). Two-step B-splines regularization method for solving an ill-posed problem of impactforce reconstruction. Journal of Sound and Vibration, vol. 297, pp. 200-214

Hu, N., Fukunaga, H., Matsumoto, S., Yan, B., Peng, X.H. (2007). An efficient approach for identifying impact force using embedded piezoelectric sensors. International Journal of Impact Engineering, vol. 34, pp. 1287-1271

Jacquelin, E., Bennani, A., Hamelin, P. (2003). Force reconstruction: analysis and regularization of a deconvolution problem. Journal of Sound and Vibration, vol. 265, pp. 81-107

Kroupa, T., Zemčík, R., Laš, V. (2011) Improved non-linear stress-strain relation for carbon-epoxy composites and identification of material parameters. Journal of composite materials, 45(9), pp. 10451057

Kudu, T., Das, S., Martin, S.A., Jata, K.V. (2008). Locating point of impact in anisotropic fiber reinforced composite plates. Ultrasonics, vol. 48, pp. 193-201

Laš, V., Zemčík, R. (2008). Progressive Damage of unidirectional composite panels. Journal of Composite Materials, vol. 42, no. 1, pp. 25-44

LeClerc, J.R., Worden, K., Staszewski, W.J., Haywood, J. (2007). Impact detection in an aircraft composite panel - A neural-network approach. Journal of Sound and Vibration, vol. 299, pp. 672-682

Martin, M.T., Doyle, J.F. (1996). Impact force identification from wave propagation responses. International Journal of Impact Engineering, vol. 18, pp. 65-77

Park, J., Chang, F.K. (2005). System identification method for monitoring impact events.Proc. SPIE 5758, pp. 189-200

Sekine, H., Atobe, S. (2009). Identification of locations and force histories of multiple point impacts on composite isogrid-stiffened panels. Composite Structures, vol. 89, pp. 1-7

Seydel, R., Chang, F.K. (2001). Impact identification of stiffened composite panels: I. System development, Smart Materials and Structures, vol. 10, pp. 354-369
Seydel, R., Chang, F.K. (2001). Impact identification of stiffened composite panels: II. Implementation studies, Smart Materials and Structures, vol. 10, pp. 370-379

Yan, G., Zhou, L. (2009). Impact load identification of composite structure using genetic algorithms. Journal of Sound and Vibration, vol. 319, pp. 869-884

Zhang, B., Zhang, J., Wu, Z., Du, S. (2008). A load reconstruction model for advanced grid-stiffened composite plates. Composite Structures, vol. 82 , pp. 600-608 